\title{
First Order Deformations of Lie Algebra Representations, E (3) and Poincaré Examples
}

\author{
Monique Levy-Nahas and Roland Seneor \\ Centre de Physique Théorique de l'Ecole Polytechnique, Paris
}

Received February 15, 1968

\begin{abstract}
A classification of "first order" deformations of Lie algebra representations by the use of a cohomology group is studied. A method is proposed for calculating this group for the case of algebras which are semi-direct products. The role of unitarity of the representations is exhibited. Applications are made for the Poincaré and $E(3)$ algebras.
\end{abstract}

Up to now, only the "first order" deformations of Lie algebra representations (connected or not with a deformation of the Lie algebra itself), seem to allow some possibilities of classification.

We recall in part $I$, how this is achieved by the introduction of a cohomology group $H^{1}(\mathscr{G}, L(V)$ ) where $V$ is the (possibly infinite-dimensional) representation space. When $\mathscr{G}$ is a semi-direct product $K . T$ of a semi-simple and compact algebra $K$ by an abelian ideal $T$, a general method can be used to determine this group $H^{2}$.

The procedure is exposed in part II; it is nearly the same as that which may be used for the computation of the finite dimensional representations of such algebras [1]. The application to the motion algebra $E(3)$ is straightforward, if one considers only the deformations leaving the rotation subalgebra and it representation fixed. For the Poincare algebra we shall see, using the "Lorentz basis" that the same method can be applied (even with a non compact $K$ ).

In all the cases, we do not claim that the method used here is completely rigourous for the infinite dimensional representations - since topological questions should be discussed in that case - nevertheless we think it has at least an heuristic value.

Our main result is that the dimension of various interesting cohomology groups $H^{1}(\mathscr{G}, L(V))$, restricted in order to produce unitary deformations, is one on $R$. This is true for $S L(2, R), V$ being a representation space for the continuous series, for $E(3)$ and the Poincare algebras, with the representations $[m, s], m>0$. It results for instance in the Poincare case that a deformation of such a representation $[m, s], m>0$ with a fixed algebra can always change the mass, the spin being "rigid". 
It results also that a "first order" deformation of the representation associated to a deformation of the algebra ${ }^{1}$ (Poincaré $\rightarrow$ De Sitter etc.) has always the general form:

$$
\begin{aligned}
\varphi_{t}\left(\mathrm{~K}_{i}\right) & =\varphi_{0}\left(\mathrm{~K}_{i}\right) ; \quad \mathrm{K}_{i} \in \mathrm{K} \\
\varphi_{t}\left(T_{i}\right) & =\varphi_{0}\left(T_{i}\right)+t\left[A, \varphi_{0}\left(T_{i}\right)\right] \quad \text { for } \quad T_{i} \in T
\end{aligned}
$$

of which the "Gell-Mann formula" is a particular example (and even the only "rational" example).

Some remarks about the deformations of the representations have been formulated in [2] (whose notations will be used here), and also in various articles of Hermann [3]. For the deformation of the algebras alone, we refer to $[4,5,6]$, and for the main cohomological definitions and results to [7] and [8].

\section{Some Generalities}

\section{Lie Algebras Deformations}

Let $\mathscr{G}=([], V)$ be a Lie algebra, $V$ its underlying vector space, and [ ], the Lie algebra law.

A deformation of $\mathscr{G}$ is a family of Lie algebras defined on the same space $V$, but with a law depending of a parameter $t: \mathscr{G}_{t}=\left([]_{t}, V\right)$ and such that:

$$
\mathscr{G}_{t} \underset{t \rightarrow 0}{\longrightarrow} \mathscr{G} .
$$

We always assume that it is possible to expand it, at least in some neighbourghood of $t=0$ :

$$
[x, y]_{t}=[x, y]+t F_{1}(x, y)+t^{2} F_{2}(x, y)+\cdots \quad x, y \in V .
$$

The functions $F_{i}$ have to be antisymmetric, and to verify a set of relations in order to satisfy the Jacobi condition. [These "integrability conditions" can be interpreted geometrically and can be expressed by an element of $H^{3}(\mathscr{G}, \mathscr{G})$.]

\section{Deformations of Representations}

Let $\varphi$ be a representation of $\mathscr{G} . \varphi$ is an homomorphism of $\mathscr{G}$ into a space $L(W)$. (One says also that $W$ is a $\mathscr{G}$-module.) A deformation $\varphi_{t}$ of $\varphi$, associated with a deformation $\mathscr{G}_{t}$ of $\mathscr{G}$, is a family of maps: $V \rightarrow L(W)$ such that:

$$
\begin{gathered}
\varphi_{t} \underset{t \rightarrow 0}{\longrightarrow} \varphi, \\
\forall t, \forall x, y \in V: \varphi_{t}\left([x, y]_{t}\right)=\left[\varphi_{t}(x), \varphi_{t}(y)\right]_{L(W)} .
\end{gathered}
$$

We shall also assume that the following expansion is valid:

$$
\varphi_{t}(x)=\varphi(x)+t \varphi_{1}(x)+t^{2} \varphi_{2}(x)+\cdots .
$$

A deformation is said of the first order if $\varphi_{2}=\varphi_{3}=\cdots=0$.

${ }^{1}$ Without "first order" term: $F_{1} . \mathrm{Ex}:\left[P_{\mu}^{\prime}, P_{\nu}^{\prime}\right]= \pm i t^{2} L_{\mu \nu}^{\prime}$ and $L_{\mu \nu}^{\prime}=L_{\mu \nu}$. 
Through (1.3), the functions $\varphi_{i}$ and $F_{i}$ have to satisfy a set of conditions:

$$
\sum_{\substack{p+q=n \\ p, q=0, \ldots n}} \varphi_{p}\left(F_{q}(x, y)\right)=\sum_{\substack{r+s=n \\ r, s=0, s, \ldots n}}\left[\varphi_{r}(x), \varphi_{s}(y)\right] .
$$

These "integrability conditions" can also be interpreted in different ways - in particular they are linked to the group $H^{2}(\mathscr{G}, L(W))$. In the following we shall restrict ourselves to the first order condition $(n=1)$ :

$$
\varphi_{0}\left(F_{1}(x, y)\right)=\left[\varphi_{1}(x), \varphi(y)\right]+\left[\varphi(x), \varphi_{1}(y)\right]-\varphi_{1}[x, y] \text {. }
$$

\section{Equivalence of two Sets $\left(\mathscr{G}_{t}, \varphi_{t}\right)$ and $\left(\mathscr{G}^{\prime}{ }_{t}, \varphi^{\prime}{ }_{t}\right)[2]$}

Let $\left(\mathscr{G}_{t}, \varphi_{t}\right),\left(\mathscr{G}_{t}^{\prime}, \varphi^{\prime}{ }_{t}\right)$ be two deformations of the same algebra $\mathscr{G}$ and representation $\varphi$.

We shall say they are equivalent if there exist a couple $\left(T_{t}, A_{t}\right)$ belonging to $(G L(V), G L(W))$ such that:

$$
\left\{\begin{array}{l}
T_{t}=\sum_{n=0}^{\infty} t^{n} T_{n} ; \quad A_{t}=\sum_{n=0}^{\infty} t^{n} A_{n} \\
A_{t}^{-1} \varphi_{t}\left(T_{t} x\right) A_{t}=\varphi^{\prime}{ }_{t}(x) \quad \forall x \in V .
\end{array}\right.
$$

In the particular case $T_{0}=A_{0}=1$, the condition (1.7) gives at the first order in $t$ :

$$
\varphi_{1}^{\prime}(x)-\varphi_{1}(x)=\varphi_{0}\left(T_{1} x\right)+\left[\varphi_{0}(x), A_{1}\right] .
$$

\section{Definition of $H^{1}(\mathscr{G}, L(W))$}

$W$ being a representation space for $\mathscr{G}$, there is a canonical way to put a $\mathscr{G}$-module structure onto the space $L(W)$, i. e. to define an action of $\mathscr{G}$ into $L(W)$ :

for $A \in L(W), x \in \mathscr{G}$, let $x . A$ be the element of $L(W)$ defined by:

$$
x . A=[\varphi(x), A]_{L(W)} .
$$

$Z^{1}(\mathscr{G}, L(W))$ : the 1-cocycle-elements of $Z^{1}(\mathscr{G}, L(W))$ are the functions $\psi$ of $\mathscr{G}$ into $L(W)$ satisfying the condition:

$$
\delta \psi(x, y)=0 \quad \forall x, y \in \mathscr{G}
$$

$\delta \psi$ being defined by the general formula:

$$
\delta \psi(x, y)=x \cdot \psi(y)-y \cdot \psi(x)-\psi([x, y])
$$

or more explicitly, using the definition of $x . A$ in our case (see 1.9):

$$
\delta \psi(x, y)=[\varphi(x), \psi(y)]-[\varphi(y), \psi(x)]-\psi([x, y]) .
$$

$B^{1}(\mathscr{G}, L(W))$ : the coboundaries are the functions $\psi: \mathscr{G} \rightarrow L(W)$ which may be put under the form:

$$
\psi(x)=x . A(=\delta A(x) \text { by definition }) .
$$


Here, this means:

$$
\psi(x)=[\varphi(x), A] .
$$

Using the Jacobi identity, it is easy to verify the inclusion $B^{1} \subset Z^{1}$.

The group $H^{1}(\mathscr{G}, L(W))$ is the quotient space: $Z^{1}(\mathscr{G}, L(W)) /$ $B^{1}(\mathscr{G}, L(W))$.

\section{Occurence of $H^{1}(\mathscr{G}, L(W))$ in the Deformations of Representations}

Let us first notice that the conditions (1.6) and (1.8) can now be written:

$$
\begin{aligned}
\varphi\left(F_{1}(x, y)\right) & =\delta \varphi_{1}(x, y), \\
\varphi_{1}^{\prime}(x)-\varphi_{1}(x) & =\varphi\left(T_{1} x\right)+\delta A_{1}(x) .
\end{aligned}
$$

a) Fixed Algebra Case

If one considers inly the deformations of the representations of a given Lie algebra, it means that all the $F_{i}(i \neq 0)$ defined in $(1.1)$ are zero, and the condition on $\varphi_{1}$ is, from $\left(1.6^{\prime}\right)$ :

$$
\delta \varphi_{1}(x, y)=0, \quad \text { i. e. } \quad \varphi_{1} \in Z^{1}(\mathscr{G}, L(W)) .
$$

The equivalence of two deformations $\varphi_{t}$ and $\varphi_{t}^{\prime}$ becomes now the usual equivalence of two representations; since with $T_{t}=1$ the Eq. (1.7) is: $A_{t}^{-1} \varphi_{t}(x) A_{t}=\varphi_{t}^{\prime}(x)$, and at the first order, one gets:

$$
\varphi_{1}^{\prime}(x)-\varphi_{1}(x)=\delta A_{1}(x), \quad \text { i. e. } \quad \varphi_{1}^{\prime}-\varphi_{1} \in B^{1}(\mathscr{G}, L(W)) .
$$

Then the elements $\varphi_{1}$ leading to inequivalent deformations of $\varphi$ have to belong to $H^{1}(\mathscr{G}, L(W))$.

In particular, one knows that if $H^{1}(\mathscr{G}, L(W))=0$, the representation $\varphi$ is rigid: all deformations are equivalent to the initial representation.

\section{b) Particular Lie Algebras Deformation}

In the particular case $F_{1}=0$, (but $F_{2}$ etc. $\neq 0$ ), $\varphi_{1}$ is always a cocycle. It is the usual way of deforming the Poincare algebra into the Ve Sitter algebras. However in that case, the representation is not in general rigid even with $H^{1}(\mathscr{G}, L(W))=0$, or more generally a coboundary does not give necessarily a deformation equivalent to the initial one (see 1.8'). An example is the "Gell-Mann Formula".

\section{c) Deformation with a Given Lie Algebra}

Let us assume that one has constructed two deformed representations $\varphi_{t}$ and $\varphi_{t}^{\prime}$, associated with the same Lie algebra deformation $\mathscr{G}_{t}$, and let us assume also that: $\varphi_{t}=\varphi_{t}^{\prime}\left(\bmod t^{n}\right)$, i. e. the two deformations 
are different only by terms of order $\geqq n$. Then $\varphi_{n}^{\prime}-\varphi_{n}$ is a cocycle. Moreover if $\varphi_{n}^{\prime}-\varphi_{n}$ is a coboundary, then the difference between $\varphi_{t}$ and $\varphi_{t}^{\prime}$ can be rejected to the next order.

The first part is a consequence of the conditions (1.5) which can be written: $\delta \varphi_{n}=\Sigma$ terms involving $F_{i}, F_{n}$, and $\varphi_{i}$ for $i<n$. For the second part: if $\left(\varphi_{n}^{\prime}-\varphi_{n}\right)(x)=[\varphi(x), A]$, let us define $A_{t}=e^{t^{n} A}$, then $\varphi_{t}^{\prime}$ and $A_{t} \varphi_{t} A_{t}^{-1}$ are identical modula $t^{n+1}$.

\section{The Semi-Simple Lie Algebras. One Example}

With the definition of equivalence given in $\mathrm{I}-3$, it is easy to prove the following result [2]:

If $G$ is a Lie algebra such that $H^{2}(\mathscr{G}, \mathscr{G})=0$ and $H^{1}(\mathscr{G}, L(W))=0$ for some representation $\varphi$ into a space $W$, then all the deformations $\left(\mathscr{G}_{t}, \varphi_{t}\right)$ of $(\mathscr{G}, \varphi)$ are equivalent to the initial set $(\mathscr{G}, \varphi)$.

This is the situation for a semi-simple Lie algebra, and a finite dimensional representation space $W$. Let us now show on one example, that it is no longer the case when $W$ is an infinite dimensional space.

Let us consider the deformation of $S L(2, R)$ representations, the algebra being fixed.

HERManN has proved that the discrete representation of $S L(2, R)$ is rigid (a result in agreement with the intuitive picture) [3]. With the restriction of making always a deformation into the unitary representations, a direct computation of $H^{1}$ for the continuous series (relative to the compact generator $J_{3}$ ), gives that:

$$
H^{1}\left(S L(2, R), J_{3}, L(W)\right)
$$

is a one dimensional space on $R$.

(The notation means that we require $\varphi_{1}\left(J_{3}\right)=0$ ).

This proves that the continuous series can be deformed with $J_{3}$ fixed, in essentially one direction (infinitesimally), as it can be also expected from a geometrical point of view. (Details are given in appendix A.)

\section{Determination of $\boldsymbol{H}^{\mathbf{1}}(\mathscr{G}, L(V))$}

In view of the application to the Euclidian and Poincare algebras, we shall first give a general way of constructing the group $H^{1}$, when the algebra is a semi-direct product $K . T$.

The procedure is suitable when $K$ is compact, or in a non-compact case, when the representation is finite-dimensional. However, we shall see that it applies also to the Poincaré case with an infinite dimensional representation.

$G$ being of the form $K . T$ with $K$ semi-simple, and $T$ abelian, we denote by $X$ the elements of $T$, and $Y$ those of $K$. 
Let us call $V$ the representation space, and $\varphi_{0}$ the representation of $\mathscr{G}$. $L(V)$ has the $\mathscr{G}$-module structure given by $(1-9)$.

When $V$ is a finite-dimensional space, the Serre-Hochschild theorem [9] simplifies the task, in particular by restricting the problem to the $K$-relative cohomology. (Since it gives $H^{1}(\mathscr{G}, L(V)) \cong H^{1}(T, L(V))^{K}$. However, in the simple case of $H^{1}$, it is easy to give a direct proof of that result.

Let $\varphi$ be a one-cochain: $\varphi \in L(\mathscr{G}, L(V))$. The restrictions of $\varphi$ respectively to $T$ and $K$ are denoted by $\varphi_{T}$ and $\varphi_{K}$. From the condition of cocycle (1.10), one gets:

$$
\begin{aligned}
& \varphi_{K} \in Z^{1}(K, L(V)) \\
& \varphi_{T} \in Z^{1}(T, L(V)) \\
& Y . \varphi_{T}(X)-X . \varphi_{K}(Y)=\varphi_{T}([Y, X]) \quad\left\{\begin{array}{l}
X \in T \\
Y \in K
\end{array}\right.
\end{aligned}
$$

using the notations defined by (1.9).

$K$ being semi-simple, $H^{1}(K, L(V))=0$, and $\varphi_{K}$ is also a coboundary:

$\exists A \in L(V)$ such that $\varphi_{K}(Y)=Y . A\left(=\left[\varphi_{0}(Y), A\right]=\delta A(Y)\right)$.

Now the map $\dot{\varphi}=\varphi-\delta A$ belongs to the same cohomology class than $\varphi$, and verifies:

$$
\begin{aligned}
& \dot{\varphi}_{K}(Y)=0 \quad \forall Y \in K \\
& \dot{\varphi}_{T} \in Z^{1}(T, L(V)) \\
& Y . \dot{\varphi}_{T}(X)=\dot{\varphi}_{T}([Y, X])
\end{aligned}
$$

[always with the notation (1.9)].

The two last conditions mean exactly that: $\dot{\varphi}_{T} \in H^{1}(T, L(V))^{K}$, (which is the Serre-Hochschild theorem).

Let us examine now the restrictions resulting from the two last conditions. In the following, we shall use the notation $\varphi$ instead of $\varphi_{T}, \varphi_{0}$ being always the representation of $\mathscr{G}$.

Condition c). We proceed in the same way as in [1]. Let us emphasize here that there is nothing more than the usual Wigner-Eckart theorem; in fact the principle is the following: by (c) $\varphi(X)$ is transforming under the subgroup $K$ exactly like the representation $\varphi_{0}(X)$ itself, hence it is clear that $\varphi(X)$ can allow transitions only between some given representations of $K$.

Let us formulate it like in [1]:

$T$ is a $K$-module for the adjoint representation, we assume that it is a simple module $D^{\alpha}$ (it is the case for $E(3)$ where $T \cong D^{1}$ and for the Poincaré algebra where $\left.T \cong D^{\frac{1}{2}} \frac{1}{2}\right)$.

$V$ is a $K$-module: by restricting the representation $\varphi$ to $K$. 
First we add the following supplementary assumptions:

$-K$ is compact, and $\varphi$ is a unitary representation

or $-K$ is non compact, and $\varphi$ is a finite-dimensional representation.

In both cases, the following decomposition is valid:

$$
V=\bigoplus_{i} D^{i} \quad \operatorname{dim} D^{i}<\infty,
$$

$D^{i}$ being an irreducible representation of $K$. (It comes from the semisimplicity of $K$ in the second case).

We restrict ourselves to cases of multiplicity one for each $D^{i}$ which occurs in the decomposition. (The general case can be also considered, according to [1], but with some more complicated notations, and we do not need it in our examples).

$T$ and $V$ being $K$-module, $L(V)$ is a $K$-module and therefore the space $L(T, L(V))$ has a canonical structure of $K$-module, defined by

where

$$
\begin{aligned}
& \psi \in L(T, L(V)) \\
& Y \in K
\end{aligned} \rightarrow Y . \psi \in L(T, L(V))
$$

$$
(Y . \psi)(X)=Y \cdot \psi(X)-\psi([Y, X]) \quad \begin{aligned}
& X \in T \\
& Y \in K .
\end{aligned}
$$

Elements $\psi$ such that $Y \cdot \psi=0$ are said $K$-invariants. The space of invariant elements is denoted by [7]:

$$
L(T, L(V))^{K}
$$

with these notations, the condition (c) can be read now:

$$
\varphi \in L(T, L(V))^{K} \text {. }
$$

Using the canonical isomorphisms:

$$
L(V) \cong \widetilde{V}^{*} \otimes V=\bigoplus_{i, j}\left(D^{i * \otimes D^{j}}\right) .
$$

The map $\varphi(T) \in L(V)$ can be decomposed into blocks $\varphi^{j i}(T) \in L\left(D^{i}, D^{j}\right)$ giving transitions between $D^{i}$ and $D^{j}$, and from (2.4):

$$
p^{j i} \in\left(D^{\alpha *} \otimes D^{i *} \otimes D^{j}\right)^{K} \text {. }
$$

Here the $K$-invariance means: projection of the product onto the trivial representation $D^{0}$ of $K$. Using again the canonical isomorphisms, one gets:

$$
\varphi\left(X_{\varepsilon}\right) e_{m}^{i}=\sum_{j, m^{\prime}} \varphi_{\varepsilon m^{\prime}}^{j i} e_{m^{\prime}}^{j},
$$

where $e_{m}^{i}$ is a basis of $D^{i}$, and $X_{\varepsilon} \in D^{\alpha}=T$. In other words, the $\varphi^{j i}$ are simply the Clebsch-Gordan coefficients used in the coupling of $D^{\alpha} \otimes D^{i}$ and $D^{j}$.

In the particular case of the $E(3)$ and Poincare algebras, the multiplicities of the representations appearing in the product decomposition is always one. When such a situation is realized, the space defined by 
(2.5) is one dimensional. But we already know an element of (2.5): the initial representation $\varphi_{0}$, restricted to $T$, obviously satisfies the conditions (c) and (b).

Hence, with the above assumption over the product decomposition, it results that:

$$
\varphi^{j i}=C^{j i} \varphi_{0}^{j i},
$$

where $C^{j i}$ is an arbitrary constant depending only on the representations $D^{i}$ and $D^{j}$.

Restrictions on the coefficients $C^{j i}$ come from the condition (2.2) b.

Condition b). It is the condition: $\varphi \in Z^{1}(T, L(V))$, i. e.

$$
X . \varphi\left(X^{\prime}\right)=X^{\prime} \cdot \varphi(X) \quad\left[\begin{array}{l}
\text { with the notation (1.9), or: } \\
{\left[\varphi_{0}(X), \varphi\left(X^{\prime}\right)\right]=\left[\varphi_{0}\left(X^{\prime}\right), \varphi(X)\right]}
\end{array}\right] .
$$

Let us "translate" first this condition in a langage very similar to the preceeding one.

For an arbitrary $\psi \in L(T, L(V))$ let us define the antisymmetric bilinear form, $B \in L(T \wedge T, L(V))$ :

Notation (1.9).

$$
B\left(X, X^{\prime}\right)=X \cdot \psi\left(X^{\prime}\right)-X^{\prime} \cdot \psi(X) .
$$

There is also a canonical $K$-module structure on the space $L(T \wedge T, L(V))$, it is defined by:

with :

$$
Y \in K \rightarrow Y . B \in L(T \wedge T, L(V))
$$$$
(Y . B)\left(X, X^{\prime}\right)=Y . B\left(X, X^{\prime}\right)-B\left([Y, X], X^{\prime}\right)-B\left(X,\left[Y, X^{\prime}\right]\right)
$$

$B$ being any antisymmetric bilinear form.

For the particular form of $B$ defined by (2.6), it is a simple consequence of the Jacobi identity, that:

$$
Y . B=0
$$

which can be noted, as before:

$$
B \in L(T \wedge T, L(V))^{K}
$$

We have also a decomposition:

$$
L(T \wedge T, L(V))^{K}=\bigoplus_{i, j} L\left(T \wedge T, L\left(D^{i}, D^{j}\right)\right)^{K}
$$

Let us call $\Sigma^{i j}$ the finite-dimensional space $L\left(T \wedge T, L\left(D^{i}, D^{j}\right)\right)^{K}$. A particular element of $\Sigma^{i j}$ is furnished by the representation itself:

$$
\begin{aligned}
& B_{0}^{j k i}\left(X, X^{\prime}\right)=\varphi_{0}^{j k}(X) \varphi_{0}^{k i}\left(X^{\prime}\right)-\varphi_{0}^{j k}\left(X^{\prime}\right) \varphi_{0}^{k i}(X) . \\
& B_{0}^{j k i} \in \Sigma^{i j}
\end{aligned}
$$

These forms are not independant; according to the commutation relation of $\varphi_{0}$, one has:

17 Commun. math. Phys., Vol. 9

$$
\sum_{k} B_{0}^{j k i}=0 . \quad \forall i, j
$$


Coming back to the condition (b), we write it now in more details between two spaces $D^{i}$ and $D^{j}$ :

$$
\left[\varphi_{0}(X), \varphi\left(X^{\prime}\right)\right]^{j i}-\left[\varphi_{0}\left(X^{\prime}\right), \varphi(X)\right]^{j i}=0, \quad \forall X, X^{\prime} \in T .
$$

Introducing the relation (2.6):

$$
\begin{aligned}
0= & \sum_{k}\left[C^{k i} \varphi_{0}^{j k}(X) \varphi_{0}^{k i}\left(X^{\prime}\right)-C^{j k} \varphi_{0}^{j k}\left(X^{\prime}\right) \varphi_{0}^{k i}(X)\right] \\
& -\sum_{k}\left[C^{k i} \varphi_{0}^{j k}\left(X^{\prime}\right) \varphi_{0}^{k i}(X)-C^{j k} \varphi_{0}^{j k}(X) \varphi_{0}^{k i}\left(X^{\prime}\right)\right]
\end{aligned}
$$

or:

$$
\sum_{k}\left(C^{j k}+C^{k i}\right) B_{0}^{j k i}\left(X, X^{\prime}\right)=0 \quad \forall X, X^{\prime} \in T .
$$

To sum up, the following relations result from (b):

$$
\begin{aligned}
\sum_{k}\left(C^{j k}+C^{k i}\right) B_{0}^{j k i} & =0 \\
\sum_{k} B_{0}^{j k i} & =0
\end{aligned}
$$

where $B_{0}^{j k i}$ is defined by $(2.10)$.

The space $Z^{1}(\mathscr{G}, L(V))$ is determined by the functions $\varphi \in L(\mathscr{G}, L(V))$ satisfying (2.6) and (2.13).

$B^{1}(\mathscr{G}, L(V)) . A$ coboundary is a linear function $\psi$ of $\mathscr{G}$ into $L(V)$, such that $\psi(X)=\delta A(X)=\left[\varphi_{0}(X), A\right]$ for some $A \in L(V)$.

We are only considering the $K$-relative cohomology. Hence $\psi$ is zero on $K$. Thus $A$ has to commute with $K$. The matrix blocks $A^{j i}$ are then of the form: $A^{j i}=\mu^{i} \delta^{j i}$, and the general form of a coboundary is:

$$
\psi^{j i}=\left(\mu^{i}-\mu^{j}\right) \varphi_{0}^{j i} .
$$

\section{Some Examples}

1. Euclidian Group $E_{3}=T_{3} \times S O(3)$

The algebra is formed by 6 generators:

$$
H_{+}, H_{-}, H_{3}, F_{+}, F_{-}, F_{3} .
$$

$H_{i}$ are the generators of the rotation subalgebra. We have also:

$$
\begin{aligned}
& {\left[H_{+}, F_{+}\right]=\left[H_{-}, F_{-}\right]=\left[H_{3}, F_{3}\right]=0} \\
& {\left[H_{+}, F_{-}\right]=-\left[H_{-}, F_{+}\right]=2 F_{3}} \\
& {\left[H_{+}, F_{3}\right]=-F_{+} ;\left[H_{-}, F_{3}\right]=F_{-} ;\left[F_{+}, H_{3}\right]=-F_{+} ;} \\
& {\left[F_{-}, H_{3}\right]=F_{-}}
\end{aligned}
$$

and the $F$ generators commute.

The representations space is $V=\bigoplus_{j=j_{0}}^{\infty} D^{j}$. 
We want to compute the group $H^{1}\left(E_{3}, L(V)\right)$, the $E_{3}$ representation on $V$ being given by the following action (with the notation $\varphi_{0}(X)=X$ ):

with

$$
\begin{aligned}
& H_{3} \xi_{j m}=m \xi_{j m} \text {, } \\
& H_{-} \xi_{j m}=\sqrt{(l+m)(l-m+1)} \xi_{j, m-1}, \\
& H_{+} \xi_{j m}=\sqrt{(l+m+1)(l-m)} \xi_{j, m+1} \text {, } \\
& F_{3} \xi_{j m}=C_{j} \sqrt{j^{2}-m^{2}} \xi_{j-1, m}-A_{j} m \xi_{j, m} \\
& -C_{j+1} \sqrt{(j+1)^{2}-m^{2}} \xi_{j+1, m} \\
& F_{+} \xi_{j, m}=C_{j} \sqrt{(j-m)(j-m-1)} \xi_{j-1, m+1} \\
& -A_{j} \sqrt{(j-m)(j+m+1)} \xi_{j, m+1} \\
& +C_{j+1} \sqrt{(j+m+1)(j+m+2)} \xi_{j+1, m+1} \\
& F_{-} \xi_{j, m}=-C_{j} \sqrt{(j+m)(j+m-1)} \xi_{j-1, m-1} \\
& -A_{j} \sqrt{(j+m)(j-m+1)} \xi_{j, m-1} \\
& -C_{j+1} \sqrt{(j-m+1)(j-m+2)} \xi_{j+1, m-1} \text {, }
\end{aligned}
$$

$$
A_{j}=\frac{a}{j(j+1)} \quad C_{j}=\frac{b}{j} \sqrt{\frac{j^{2}-j_{0}^{2}}{4 j^{2}-1}}, \quad b=\frac{\varepsilon i a}{j_{0}}
$$

and $a$ is a real number.

(This representation is easily obtained by contraction of the representations of $S L(2, C)$ given in Gelfand-Minlos-Shapiro).

a) "Unrestricted" $H^{1}\left(E_{3}, S O(3), L(V)\right)$

We shall consider the deformation "around" the rotation part: i. e. leaving fixed the representation of $S O(3)$. It corresponds to a computation of a $S O(3)$ relative cohomology group, denoted by $H^{1}\left(E_{3}, S O(3)\right.$, $L(V)$ ) (its elements are required to be zero on $S O(3)$ ). From (II) (2.1), it is the general situation when $V$ is a finite dimensional space. By "unrestrictive cohomology" we want to emphasize that there is no supplementary conditions on the cochains. In particular, we do not make any condition of unitarity in that part. The deformation obtained with this elements of $H^{1}$ can lead to non-unitary representations.

Let $\varphi$ be an element of $H^{1}\left(E_{3}, S O(3), L(V)\right)$. We have

$$
\varphi\left(H_{+}\right)=\varphi\left(H_{-}\right)=\varphi\left(H_{3}\right)=0 .
$$

1) From condition (2.6), we have only 3 constants to introduce; it is convenient to choose the following ones

$$
\begin{aligned}
\varphi^{j-1, j} & =\frac{\alpha_{j}}{C_{j}} \varphi_{0}^{j-1, j} \\
\varphi^{j, j} & =-\frac{\beta_{j}}{A_{j}} \varphi_{0}^{j, j} \\
\varphi^{j+1, j} & =-\frac{\gamma_{j}}{C_{j+1}} \varphi_{0}^{j+1, j} .
\end{aligned}
$$


For example, one has:

$$
\begin{aligned}
\varphi\left(F_{3}\right) \xi_{j, m}= & \alpha_{j} \sqrt{j^{2}-m^{2}} \xi_{j-1, m}+\beta_{j} m \xi_{j, m} \\
& +\gamma_{j} \sqrt{(j+1)^{2}-m^{2}} \xi_{j+1, m} .
\end{aligned}
$$

2) Let us write explicitly the condition (2.2) c in that case:

$$
\begin{array}{ll}
2^{\prime} & \left(\beta_{j} C_{j+1}+A_{j} \gamma_{j}\right) j=\left(\gamma_{j} A_{j+1}+C_{j+1} \beta_{j+1}\right)(j+2) \\
2^{\prime \prime} & \left(\alpha_{j} A_{j-1}-C_{j} \beta_{j-1}\right)(j-1)=\left(A_{j} \alpha_{j}-\beta_{j} C_{j}\right)(j+1) \\
2^{\prime \prime \prime} & \left(\gamma_{j-1} C_{j}-C_{j} \alpha_{j}\right)(2 j-1)=2 \beta_{j} A_{j}+\left(\gamma_{j} C_{j+1}-C_{j+1} \alpha_{j+1}\right)(2 j+3) .
\end{array}
$$
From

$2^{\prime}$ and $2^{\prime \prime}$ give

$$
A_{j} j=A_{j+1}(j+2)
$$

therefore

$$
\beta_{j} j=\beta_{j+1}(j+2),
$$

$$
\beta_{j}=\frac{\varrho}{j(j+1)}
$$

$\varrho$ is a constant (possibly complex).

With $r_{j}=\gamma_{j-1}-\alpha_{j}$ we are left to solve

with

$$
(2 j+1) C_{j} r_{j}=\frac{2 \varrho a}{j^{2}(j+1)^{2}}+(2 j+3) C_{j+1} r_{j+1},
$$

we get

$$
C_{j} r_{j}(2 j-1)(2 j+1)=\sigma_{j},
$$

or

$$
\sigma_{j}=\frac{2 \varrho a(2 j+1)}{j^{2}(j+1)^{2}}+\sigma_{j+1}
$$

thus

$$
\sigma_{j}-\frac{2 \varrho a}{j^{2}}=C^{\mathrm{te}}=C
$$

$$
r_{j}=\frac{j}{b} \frac{1}{\sqrt{\left(4 j^{2}-1\right)\left(j^{2}-j_{0}^{2}\right)}}\left(C+\frac{2 \varrho a}{j^{2}}\right)=\gamma_{j-1}-\alpha_{j} .
$$

Thus the general solution of the system is

$$
\begin{aligned}
\beta_{j}=\frac{\varrho}{j(j+1)} & =-\lambda A_{j} \quad \text { with }-\lambda a=\varrho \\
\gamma_{j-1} & =-\lambda C_{j}+k_{j} \\
\alpha_{j} & =\lambda C_{j}+k_{j}^{\prime}
\end{aligned}
$$


Therefore for $F$ operators:

$$
\left[A, \varphi_{0}(F)\right]=\left(\mu_{j-1}-\mu_{j}\right) C_{j}[] \xi_{j-1, m^{\prime}}-\left(\mu_{j+1}-\mu_{j}\right) C_{j+1}[] \xi_{j+1, m^{\prime}}
$$

(where we have omitted the explicit expression of the rotation ClebshGordan coefficients). From the existence of a lower dimension space $D^{j_{0}}$ in $V$, we have to ask that:

or:

$$
\alpha_{j_{0}}=\gamma_{j_{0}-2}=0
$$

$$
r_{j_{0}}=0 \text {. }
$$

The only solution for the constant $C$ independant of $j$ ) is:

$$
C=-\frac{1}{j_{0}^{2}} \text {. Then } r_{j}=\frac{2 \varrho a}{b} j \sqrt{\frac{j^{2}-j_{0}^{2}}{4 j^{2}-1}}=-2 \lambda C_{j}\left(\text { with } \lambda=-\frac{\varrho}{a}\right) \text {. }
$$

From (3.4):

$$
\begin{aligned}
& \alpha_{j}=\lambda C_{j}+k_{j} \\
& \gamma_{j-1}=-\lambda C_{j}+k_{j} .
\end{aligned}
$$

Now, we will show that $k_{j}$ is a matrix element of a coboundary i. e. from (3.6) we look for arbitrary complex numbers $\mu_{j}$ such that:

A solution is:

$$
\left\{\begin{array}{c}
\left(\mu_{j-1}-\mu_{j}\right) C_{j}=k_{j} \\
-\left(\mu_{j+1}-\mu_{j}\right) C_{j+1}=k_{j+1} .
\end{array}\right.
$$

We have thus shown that:

$$
\left.\begin{array}{l}
\mu_{j}=-\sum_{j_{0}+1}^{j} \frac{k_{j}}{C_{j}} \\
\mu_{j_{0}}=0 .
\end{array}\right\}
$$

$$
\varphi(F)=\lambda \varphi_{0}(F)+\left[A, \varphi_{0}(F)\right]
$$

where $\lambda$ is a complex number, $A$ an operator defined by (3.7) and (3.5), or equivalently:

$$
\operatorname{dim} H^{1}\left(E_{3}, S O(3), L(V)\right)=1 \quad \text { on } \mathbb{C} .
$$

$(S O(3)$ means we consider only the $S O(3)$ relative cohomology: it corresponds to leave $S O(3)$ fixed during the deformation).

b) Restricted $H^{1}\left(E_{3}, S O(3), L(V)\right)$

To consider the deformation of $E(3)$ representations into unitary representations of the group (i. e. with hermitic operators $\varphi(F)$ ) $\left(F_{3}=F_{3}^{+},\left(F^{+}\right)^{+}=F^{-}\right)$, we are led to introduce some restrictions on the $\varphi(F)$. They are:

$$
\begin{aligned}
& \alpha_{j}=\bar{\gamma}_{j-1}, \\
& \beta_{j}=\bar{\beta}_{j} .
\end{aligned}
$$


From (3.10), one gets that $\varrho$ is a real number. (See (3.4)) and from (3.9), since $C_{j}$ is pure imaginary, and $\lambda$ real, that $k_{j}$ has to be real. (The $\left(\mu_{j}\right)$ are then pure imaginary). Introducing these restrictions in the preceding results, one gets:

$$
\operatorname{dim} H_{r}^{1}\left(E_{3}, S O(3), L(V)\right)=1 \quad \text { on } \mathbb{R} \quad(r=\text { restricted }) .
$$

Remark. For the deformation of representation of $E(3)$, the algebra being fixed, the picture associated with these results is very analogous to those which appears in the $S L(2, R)$ case (see appendix). The discrete casimir is left unchanged, the continuous one is translated along a real axis for the unitary deformed representations.

\section{The Poincaré Group}

In attempt to extend the above method (part II), it is natural to look for an expression of the Poincaré's unitary representations in a basis where the Lorentz subalgebra is diagonal. This procedure seems particularly suitable for a deformation leaving the homogeneous Lorentz part fixed. In the following we restrict ourselves to that kind of deformations only. We shall use the Lorentz basis form of the representations.

\section{a) Spin Zero Case}

Let us consider first a representation $[m, s]$ with $m>0$ and $S=0$. Restricted to $S L(2, C)$, it has the following reduction [Joos]:

(a) $D^{[m, 0]}=\int_{0}^{\infty} d \lambda \mathscr{D}^{0, i \lambda}$.

(Denoting the unitary representations of $S L(2, C)$ in the usual way: $\left.\mathscr{D}^{0, i \lambda}\right)$.

In [10], it is shown that it is possible to transform the usual Poincaré's representation, in such a way that the translation operators act like ladder operators between the non-unitary representations of $S L(2, C)$.

For example (dropping the index $j_{0}=0$ )

$$
\begin{aligned}
& \text { (b) } \varphi_{0}\left(P_{0}\right)|j m ; \lambda\rangle=\frac{M}{2[\lambda(\lambda-i)]^{1 / 2}}[(j-i \lambda)(j+i \lambda+1)]^{1 / 2}|j m ; \lambda-i\rangle \\
& +\frac{M}{2[\lambda(\lambda+i)]^{1 / 2}}[(j+i \lambda)(j-i \lambda+1)]^{1 / 2}|j m ; \lambda+i\rangle .
\end{aligned}
$$

And there is at least a "formal" equivalence between the direct integral (a) and direct sum:

$$
\bigoplus_{n=-\infty}^{+\infty} \mathscr{D}^{0, i \lambda+n}
$$

[Since we can reconstruct the usual representation, from expression like (b)]. 
It is also in a similar sense (not very rigourous mathematically ...) that we shall apply now the results of the section II. The dimension one for spaces like (2.5) or (2.9) comes from computations made in [10].

From (2.6), a cocycle is a transition operator between $\mathscr{D}^{0, i \lambda} \rightarrow \mathscr{D}^{0, i \lambda \pm 1}$, it corresponds to two sorts of coefficients $C^{\lambda+i, \lambda}$ and $C^{\lambda-i, \lambda}$. (2.13) gives only one non trivial relation on the symmetric part $S$ of these coefficients:

$$
S^{\lambda+i, \lambda}=S^{\lambda, \lambda+i}=C^{\mathrm{te}}=a
$$

where $\mathrm{a}$ is an arbitrary (possibly complex) number.

The antisymmetric part $A^{j, i}$ of $C^{j, i}$ can always be put under the form: $\mu^{i}-\mu^{j}$, which here also corresponds to a coboundary. Let us take for example:

Then

$$
\begin{aligned}
b(p) & =\sum_{n=1}^{p} A^{\lambda+(n-1) i, \lambda+n i} \\
b(-p) & =\sum_{n=1}^{p} A^{\lambda-n i, \lambda-(n-1) i} \\
b(0) & =0 .
\end{aligned}
$$

Finally, every cocycle can be put under the form:

$$
\varphi^{p+1, p}=a \varphi_{0}^{p+1, p}+(b(p)-b(p+1)) \varphi_{0}^{p+1, p},
$$

where we had written $p$ for $\lambda+i p$.

Restricted deformations into unitary representations: it is not possible to express directly in a non ambiguous way the unitarity of the group representations in the "Lorentz basis". However, using the explicit form of the hermitian operator $\varphi_{0}\left(P_{0}\right)$ we shall admit that a condition of hermiticity for $\varphi_{1}\left(P_{0}\right)$ is:

$$
C^{\lambda, \lambda+1}=\overline{C^{\lambda, \lambda-1}}
$$

With this condition, $a$ is now a real number.

Therefore from (3.13) $\operatorname{dim} H^{1}=1$ (on $R$ ).

\section{b) Representation $m>0, S \neq 0$}

Four types of transition are allowed now. They are:

$$
\left(j_{0}, \lambda\right) \rightarrow\left(j_{0} \pm 1, \lambda\right)
$$

or

$$
\left(j_{0}, \lambda \pm i\right) \text {. }
$$

From (2.6), the matrix elements of the cocycle are proportionnal to those of the initial representation:

$$
\begin{aligned}
& \varphi_{1}^{j_{0} \pm 1, \lambda ; j_{0} \lambda}=C_{\lambda}^{j_{0} \pm 1 ; j_{0}} \varphi_{0}^{j_{0} \pm 1, \lambda ; j_{0} \lambda} \\
& \varphi_{1}^{j_{0}, \lambda \pm i ; j_{0} \lambda}=C_{j_{0}}^{\lambda+i, \lambda} \varphi_{0}^{j_{0}, \lambda \pm i, j_{0} \lambda} .
\end{aligned}
$$


The conditions (2.13) on the coefficients are of two sorts, according to the final state $\left(j_{0}^{\prime}, \lambda^{\prime}\right)$ is different or not from the initial state $\left(j_{0}, \lambda\right)$.

a) $\left(j_{0}^{\prime}, \lambda^{\prime}\right) \neq\left(j_{0}, \lambda\right)$.

The situation can be pictured by:

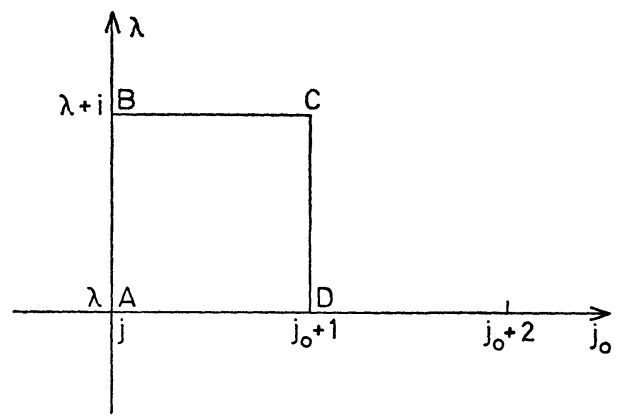

Fig. 1

Starting from $A, B$ is a possible final state, and two ways are allowed: $A B C$ and $A D C$.

The 2 conditions (2.13) may then be written (with obvious notations)

$$
C^{C B}+C^{B A}=C^{C D}+C^{D A} .
$$

Starting from $C$ and going to $A$, one sees that the symmetric part and the antisymmetric part of $C^{A B}$ satisfy independently this condition. Let us examine first the symmetric part of the coefficient $S^{A B}$. If we start from $B$ to $D$, we have:

$$
S^{D C}+S^{C B}=S^{D A}+{ }^{A B}
$$

with (3.16), it gives:

or more explicitly:

$$
\left\{\begin{array}{l}
S^{A B}=S^{D C} \\
S^{D A}=S^{C B}
\end{array}\right.
$$

$$
\begin{aligned}
S_{j_{0}+1}^{\lambda+i, \lambda} & =S_{j_{0}}^{\lambda+i, \lambda} \\
S_{\lambda}^{j_{0}+1, j_{0}} & =S_{\lambda+i}^{j_{0}+1, j_{0}}
\end{aligned}
$$

i. e. the symmetric part are independent of the "fixed index"

$$
\begin{aligned}
S_{j_{0}}^{\lambda+i, \lambda} & =S^{\lambda+i, \lambda} \\
S_{\lambda}^{j_{0}+1, j_{0}} & =S^{j_{0}+1, j_{0}} .
\end{aligned}
$$

The antisymmetric part $A^{A B}$ can easily be put under the form of a difference $\lambda^{A}-\lambda^{B}$. It is enough to observe from (3.16) that it obeys a Chasles relation (and to proceed in the same way as in 3.12). 
B) $\left(j_{0}^{\prime}, \lambda^{\prime}\right)=\left(j_{0}, \lambda\right)$.

The situation is a little more complicated since four ways are now allowed:

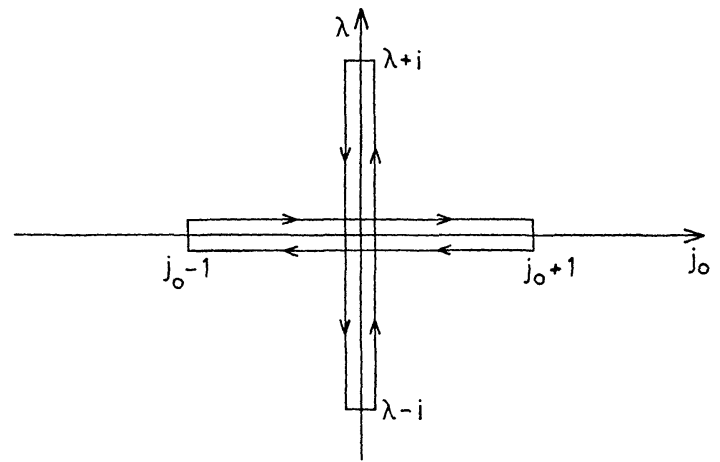

Fig. 2

We have to compute the forms $B_{0}$ defined by (2.10). It is enough to compute only the diagonal term. We use the following notation:

$$
j_{0}, \lambda\left\langle j m\left|B^{j_{0}+1}\left(P_{0}, P_{3}\right)\right| j m\right\rangle_{j_{0}, \lambda}=B^{j_{0}+1} \text {. }
$$

Up to factor, they are:

$$
\begin{aligned}
& B^{j_{0}+1}=-i \lambda\left(j-j_{0}\right)\left(j+j_{0}+1\right) \beta_{j 0} \\
& B^{j_{0}-1}=+i \lambda\left(j+j_{0}\right)\left(j-j_{0}+1\right) \beta_{j_{0}-1} \\
& B^{\lambda+i}=j_{0}(j+i \lambda)(j-i \lambda+1) \alpha_{\lambda} \\
& B^{\lambda-i}=-j_{0}(j-i \lambda)(j+i \lambda+1) \alpha_{i-i} .
\end{aligned}
$$

We use the notations of [10] for the expression of the representations. For example:

$$
\begin{aligned}
P_{0}|j m\rangle_{j_{0} \lambda}= & C_{0 \lambda}^{j_{0}+1, j_{0}}\left[\left(j-j_{0}\right)\left(j+j_{0}+1\right)\right]^{1 / 2}|j m\rangle_{j_{0}+1, \lambda} \\
& +C_{0 \lambda}^{j_{0}-1, j_{0}}\left[\left(j+j_{0}\right)\left(j-j_{0}+1\right)\right]^{1 / 2}|j m\rangle_{j_{0}-1, \lambda} \\
& +C_{0 j_{0}}^{\lambda+i, \lambda}[(j+i \lambda)(j-i \lambda+1)]^{1 / 2}|j m\rangle_{j_{0}, \lambda+i} \\
& +C_{0 j_{0}}^{\lambda-i, \lambda}[(j-i \lambda)(j+i \lambda+1)]^{1 / 2}|j m\rangle_{j_{0}, \lambda-i}
\end{aligned}
$$

and

$$
\begin{array}{r}
C_{j_{0}}^{\lambda, \lambda+i} C_{j_{0}}^{\lambda+i, \lambda}=\alpha_{\lambda} \\
C_{\lambda}^{j_{0}+1, j_{0}} C_{\lambda}^{j_{0}, j_{0}+1}=\beta_{j_{0}}
\end{array}
$$

$\alpha_{\lambda}$ and $\beta_{j_{\mathrm{e}}}$ being explicitly computed in [10]. For a representation $[m, S]$ :

$$
\alpha_{\lambda}=\frac{m^{2}}{4} \frac{(s+i \lambda)(s-i \lambda+1)}{\left(j_{0}+i \lambda\right)\left(j_{0}-i \lambda+1\right)\left(j_{0}-i \lambda\right)\left(j_{0}+i \lambda-1\right)},
$$


$\beta_{j_{0}}$ is obtained by changing $j_{0} \leftrightarrow-i \lambda$.

Let us put now

From the conditions $(2.13)$ :

$$
\begin{aligned}
& \alpha_{\lambda}^{\prime}=S^{\lambda, \lambda+i} \alpha_{\lambda} \\
& \beta_{j_{0}}^{\prime}=S^{j_{0}, j_{0}+1} \beta_{j_{0}} .
\end{aligned}
$$

$S^{\lambda, \lambda+i} B_{0}^{\lambda+i}+S^{\lambda, \lambda-i} B_{0}^{\lambda-i}+S^{j_{0}, j_{0}+1} B_{0}^{j_{0}+1}+S^{j_{0}, j_{0}-1} B_{0}^{j_{0}-1}=0$

or (using the independence of $j$ of the constants).

The solution of this equation is

$$
\begin{aligned}
j_{0}\left(\alpha_{\lambda}^{\prime}-\alpha_{\lambda-i}^{\prime}\right) & =i \lambda\left(\beta_{j_{0}}^{\prime}-\beta_{i_{0}-1}^{\prime}\right) \\
i \lambda\left(\alpha_{\lambda}^{\prime}-\alpha_{\lambda-i}^{\prime}\right) & +j_{0}\left(\beta_{j_{0}}^{\prime}-\beta_{j_{0}-1}^{\prime}\right) \\
& +\left(\alpha_{\lambda}^{\prime}+\alpha_{\lambda-i}^{\prime}\right)+\left(\beta_{j_{0}}^{\prime}+\beta_{j_{0}-1}^{\prime}\right)=0 .
\end{aligned}
$$

$$
\begin{aligned}
\alpha_{\lambda}^{\prime} & =\frac{\lambda(\lambda+i) \alpha-\gamma}{\left(j_{0}^{2}+\lambda^{2}\right)\left(j_{0}^{2}+(\lambda+i)^{2}\right)} \\
\beta_{i_{0}}^{\prime} & =\frac{-j_{0}\left(j_{0}+1\right) \alpha-\gamma}{\left(j_{0}^{2}+\lambda^{2}\right)\left(\left(j_{0}+1\right)^{2}+\lambda^{2}\right)},
\end{aligned}
$$

where $\alpha$ and $\gamma$ are two constants $(\alpha, \gamma \in C)$. Using the explicit form of $\alpha_{\lambda}$ and $\beta_{j_{0}}$, one gets finally:

$$
\begin{aligned}
S^{\lambda, \lambda+i} & =\frac{4}{m^{2}} \frac{\lambda(\lambda+i) \alpha-\gamma}{(s+i \lambda)(s-i \lambda+1)} \\
S^{j_{0}, j_{0}+1} & =-\frac{4}{m^{2}} \frac{j_{0}\left(j_{0}+1\right) \alpha+\gamma}{\left(s-j_{0}\right)\left(s+j_{0}+1\right)} .
\end{aligned}
$$

The initial representation being of spin $S, j_{0}$ has to belong to $[-S,+S]$. The only way of eliminate the poles for $s=j_{0},-j_{0}-1$ in $(3.26)^{2}$ is to put: $\gamma=-S(S+1) \alpha$. But then:

$$
S^{\lambda, \lambda+i}=\frac{4 \alpha}{m^{2}}=S^{j_{0}, j_{0}+1}=\alpha^{\prime}
$$

We have then:

$$
\varphi_{1}\left(P_{\mu}\right)=\alpha^{\prime} \varphi_{0}\left(P_{\mu}\right)+\left[A, \varphi_{0}\left(P_{\mu}\right)\right]
$$

where $\alpha^{\prime}$ is a possible complex number.

Exactly as in the Euclidian case, we do not have made until now any restriction about the hermiticity. The condition that $\varphi_{1}$ is an hermitian operator is equivalent to the condition $\alpha^{\prime}$ is a real number and $A$ an antihermitian operator (in the impulsion basis).

\section{c) Zero-Mass Representation and Discrete Helicity}

The reduction on $S L(2, C)$ have been given in [10] and in [11]. There is only one possible transition from $\left(j_{0}, \lambda\right)$ to $\left(j_{0}, \lambda-i\right)$ or $\left(j_{0}, \lambda+i\right)$, depending upon the sign + or - of the helicity, therefore one type of

\footnotetext{
${ }^{2}$ In fact in $S^{j_{0} j_{0}+1} \cdot C^{j_{0} j_{0}+1}$.
} 
coefficient:

$$
\varphi_{1}^{\lambda-i, \lambda}\left(p_{\mu}\right)=C^{\lambda-i, \lambda} \varphi_{0}^{\lambda-i, \lambda}\left(P_{\mu}\right) .
$$

In that case, the conditions (2.13) are always trivially satisfied: there is no condition on $C^{\lambda-i, \lambda}$.

Remark. A simple change of basis in the space $V=\oplus D^{j_{0}, \lambda+\text { in }}$ transform $\varphi_{1}$ into $\varphi_{0}$.

\section{d) Continuous Spin and Zero-Mass Representation}

With $W^{2}=-\zeta^{2} I, \zeta$ continuous, the coefficients $\alpha_{\lambda}$ and $\beta_{j_{0}}$ of the initial representation are:

$$
\begin{aligned}
\alpha_{\lambda} & =\frac{\zeta^{2}}{4\left(j_{0}^{2}+\lambda^{2}\right)\left(j_{0}^{2}+(\lambda+i)^{2}\right)}, \\
\beta_{j_{0}} & =\frac{\zeta^{2}}{\left.4\left(j_{0}^{2}+\lambda^{2}\right)\left(j_{0}+1\right)^{2}+\lambda^{2}\right)} .
\end{aligned}
$$

Four transitions are allowed in that case, and the computation is exactly the same as in the case $S \neq 0, m>0$.

The only difference is that the domain of variation of $j_{0}$ is now $-\infty<j_{0}<+\infty$.

From (3.25) one gets for the symmetric part of the coefficient:

$$
\begin{aligned}
S^{\lambda, \lambda+i} & =\frac{4}{\zeta^{2}}(\lambda(\lambda+i) \alpha-\gamma) \\
S^{j_{0}, j_{0}+1} & =-\frac{4}{\zeta^{2}}\left(j_{0}\left(j_{0+1}\right) \alpha+\gamma\right) .
\end{aligned}
$$

Finally, we have obtained the following results: for a unitary representation $[m, S] m>0, S \geqq 0$ of the Poincaré group $\mathscr{P}$, the group $H^{1}(\mathscr{P}, L(V))$ is a one dimensional space on $C$. With the "unitary restriction", it is a one dimensional space on $R$.

In the case of the zero-mass representation, the obtained cocycle can always be transformed into the initial representation $\varphi_{0}$ by a change of basis.

In the continuous spin case, $H^{1}(\mathscr{P}, L(V))$ is a 2-dimensional space on $C$ in general.

\section{Application to the Deformations of Representations}

\section{With a Fixed Algebra}

In that case the coboundaries may always be rejected by means of a unitary transformation, and the only thing we have to consider is the deformation produced by the representative elements of $H^{1}(\mathscr{G}, L(V))$ [Cf. I. 5a].

It results from III, that the only deformations leaving the Lorentz subalgebra fixed of the Poincaré representation $[m, S] m \neq 0$ are of the 
form:

$$
\left\{\begin{array}{l}
\varphi_{t}\left(M_{\mu \nu}\right)=0 \\
\varphi_{t}\left(P_{\mu}\right)=\alpha(t) \varphi_{0}\left(P_{\mu}\right),
\end{array}\right.
$$

where $\alpha(t)=1+t a+t^{2} b+\ldots$

and correspond to a representation $\left[\mathrm{m}^{\prime}, S\right]$ with the same spin value, and with a different mass: $m^{\prime}(t)=\alpha(t) m_{0}$. Of course this is not surprising, since the rigidity of the spin is expected.

A similar result holds for the $E(3)$ algebra. For the zero mass representation, it results from III, $\mathrm{B}$, that the representation $\left[m=0, j_{0}\right]$ is rigid. But here, it has to be noticed that the condition for the Lorentz algebra to be fixed is certainly too strong. In fact, if one allows the generators $\boldsymbol{N}$ to move, $\boldsymbol{J}$ being fixed, one can easily deform the mass zero representation of helicity $S$ into a representation of mass $m \neq 0$. It follows clearly from the expressions of the generators given in [12].

\section{With a Deformation of the Algebra. The "Gell-Mann Formula"}

According to a general result of Richardson on the stability of the subalgebras, one knows that all the deformations of the algebra $K . T^{3}$ is of the following form:

the only new commutator is: and

$$
\left[X_{\alpha}, X_{\beta}\right]_{t}=\left[X_{\alpha}, X_{\beta}\right]_{0}+t F_{1}\left(X_{\alpha}, X_{\beta}\right)+\ldots \text { where } \quad X_{\alpha}, X_{\beta} \in T
$$

$$
F_{i}\left(X_{\alpha}, X_{\beta}\right) \in K
$$

Let us consider now a second order deformation $\mathscr{G}_{t}$ of the semi-direct product $\mathscr{G}=K . T$

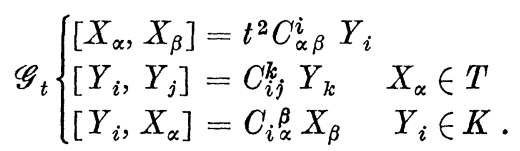

The first order deformations of the representation have already been examined with different methods by BEHREND [13] and HERMaNN [14].

With :

$$
\left.\begin{array}{l}
\varphi_{t}(Y)=\varphi_{0}(Y) \\
\varphi_{t}(X)=\varphi_{0}(X)+t \varphi_{1}(X),
\end{array}\right\}
$$

they have found that it is possible to take (with some assumptions):

$$
\varphi_{1}(X)=\left[\Delta, \varphi_{0}(X)\right]
$$

where $\Delta$ is the second order casimir of $K$, suitably "normalized". The relation thus obtained, already known in some cases in the physics litterature (for example $[15,16,17]$ ) was called the "Gell-Mann formula". For more details about that formula, we refer to Hermann articles [14].

\footnotetext{
${ }^{3} K$ is semi-simple and $T$ abelian.
} 
Let us consider the Poincaré algebra (or $E(3)$ algebra). We know from (III) that the general form of $\varphi_{1}$ is:

$$
\varphi_{1}(X)=\lambda \varphi_{0}(X)+\left[A, \varphi_{0}(X)\right],
$$

where $A$ commutes with $\varphi_{0}(K) . A$ is then determined by its matrix elements $a\left(j_{0}, \lambda\right)$, depending only on the Lorentz representation $\left(j_{0} \lambda\right)$.

In the following, we found the general form of $A$ for a deformation of a Poincaré representation $[m, S] m \neq 0$ corresponding to the following deformation:

$$
\begin{aligned}
& {\left[L_{\mu \nu}, L_{\varrho \sigma}\right]=i\left(g_{\mu \sigma} L_{\nu \varrho}-g_{\mu_{\varrho}} L_{\nu \sigma}+g_{v \varrho} L_{\mu \sigma}-g_{\nu \sigma} L_{\mu \varrho}\right.} \\
& {\left[L_{\mu \nu}, P_{\varrho}^{\prime}\right]=i\left(g_{\nu \varrho} P_{\mu}^{\prime}-g_{\mu \varrho} P_{v}^{\prime}\right)} \\
& {\left[P_{\mu}^{\prime}, P_{\varrho}^{\prime}\right]=\varepsilon i t^{2} L_{\mu \varrho} .}
\end{aligned}
$$

$\varepsilon=+1$ gives a deformation into $S O(3,2)$, and

$\varepsilon=-1$ gives a deformation into $S O(4,1)$.

We have used the notation

$$
\begin{aligned}
& P_{\mu}^{\prime}=\varphi_{t}\left(P_{\mu}\right) ; \quad L_{\mu \nu}=\varphi_{0}\left(L_{\mu \nu}\right)=\varphi_{t}\left(L_{\mu_{2}}\right) \\
& P_{\mu}=\varphi_{0}\left(P_{\mu}\right) .
\end{aligned}
$$

We have to study the second-order condition of commutation, i. e.:

$$
\left[\varphi_{1}\left(P_{\mu}\right), \varphi_{1}\left(P_{v}\right)\right]=\varepsilon i L_{\mu v},
$$

where $\varphi_{1}$ is given by (4.5):

$$
\varphi_{1}\left(P_{\mu}\right)=\lambda P_{\mu}+\left[A, P_{\mu}\right] .
$$

a) Spin Zero Case

$j_{0}=0$, the matrix elements of $A$ are only functions of $\lambda$. (4.7) gives only a condition on the coboundary part:

Let us define:

$$
\left[\left[A, P_{\mu}\right],\left[A, P_{\nu}\right]\right]=\varepsilon i L_{\mu \nu} .
$$

we have

$$
\sigma(\lambda)=a(\lambda+i)-a(\lambda)
$$

$$
\begin{aligned}
& {\left[A, P_{\mu}\right]^{\lambda+i, \lambda}=\sigma(\lambda) P_{\mu}^{\lambda+i, \lambda}} \\
& {\left[A, P_{\mu}\right]^{\lambda-i, \lambda}=-\sigma(\lambda-i) P_{\mu}^{\lambda-i, \lambda}}
\end{aligned}
$$

and (4.8) is equivalent to:

$$
\begin{aligned}
& -\sigma^{2}(\lambda)\left(P_{\mu}^{\lambda, \lambda+i} P_{\nu}^{\lambda+i, \lambda}-P_{\nu}^{\lambda, \lambda+i} P_{\mu}^{\lambda+i, \lambda}\right) \\
& -\sigma^{2}(\lambda-i)\left(P_{\mu}^{\lambda, \lambda-i} P_{\nu}^{\lambda-i, \lambda}-P_{\nu}^{\lambda, \lambda-i} P_{\mu}^{\lambda-i, \lambda}\right)=\varepsilon i L_{\mu \nu}
\end{aligned}
$$

(the other parts are identically verified).

With the notations introduced in part II:

$$
\sigma^{2}(\lambda) B_{0}^{\lambda+i}\left(P_{\mu}, P_{v}\right)+\sigma^{2}(\lambda-i) B_{0}^{\lambda-i}\left(P_{\mu}, P_{v}\right)=-\varepsilon i L_{\mu \nu} .
$$


It is enough to examine one matrix element of the preceding form. Let us choose (see [10]):

$$
\begin{aligned}
& \langle\lambda, j+1, m| B_{0}^{(\lambda+i)}\left(P_{0}, P_{3}|\lambda, j, m\rangle\right. \\
= & -2\left[\frac{(j+1)^{2}-m^{2}}{(2 j+1)(2 j+3)}\right]^{1 / 2} \alpha_{\lambda}\left[(j+1)^{2}+\lambda^{2}\right]^{1 / 2}(\lambda+i),
\end{aligned}
$$

we have

$$
\left\langle\lambda, j+1, m\left|L_{03}\right| \lambda, j, m\right\rangle=\left[\frac{(j+1)^{2}-m^{2}}{(2 j+1)(2 j+3)}\right]^{1 / 2}\left((j+1)^{2}+\lambda^{2}\right)^{1 / 2}
$$

and we know from the commutation of the $P_{\mu}$, that:

$$
B_{0}^{\lambda+i}+B_{0}^{\lambda-i}=0 \text {. }
$$

Finally one gets from (4.10):

$$
\left[\sigma(\lambda)^{2}-\sigma(\lambda-i)^{2}\right]=\frac{\varepsilon \cdot i}{2 \alpha_{\lambda}(\lambda+i)} .
$$

In the spin zero case

therefore

$$
\alpha_{\lambda}=\frac{m^{2}}{4 \lambda(\lambda+i)} .
$$

$$
\sigma(\lambda)^{2}-\sigma(\lambda-i)^{2}=\frac{2}{m^{2}} \varepsilon i \lambda,
$$

the solution of (4.11) is easy to obtain in 2 steps:

$$
\sigma^{2}(\lambda)=\frac{\varepsilon}{m^{2}}\left(\lambda^{2}+i \lambda+\gamma\right)
$$

$\gamma$ is an arbitrary constant

$$
\text { then: } \quad a(\lambda+i)-a(\lambda)= \pm \frac{\sqrt{\bar{\varepsilon}}}{m}\left(\lambda^{2}+i \lambda+\gamma\right)^{1 / 2} .
$$

The general solution for $a(\lambda)$ can be deduced from (4.13). It is a sum of square roots, and furnishes a dependence in $\lambda$ which is in general irrational. i. e. $A$ is an irrational function of $\boldsymbol{M}^{2}-\boldsymbol{N}^{2}$. The only simple (and rational) case corresponds to $\gamma=-1 / 4$, i.e.:

$$
a(\lambda+i)-a(\lambda)= \pm \frac{\sqrt{\varepsilon}}{m}\left(\lambda+\frac{i}{2}\right) .
$$

This is easily solved:

$$
a(\lambda)=\mp \frac{\sqrt{\varepsilon}}{2 m} i\left(1+\lambda^{2}\right)+a \text { constant }
$$

Or equivently:

$$
A= \pm \frac{i \sqrt{\varepsilon}}{2 m}\left(\boldsymbol{N}^{2}-\boldsymbol{M}^{2}\right)+\text { constant },
$$

which corresponds to the "Gell-Mann Formula".

[The hermitic solution corresponds to $\varepsilon=+1$, i. e. to a deformation into $S O(3,2)]$. 
b) Initial Representation: $[m, S] m>0, S \neq 0$

In the diagonal part of the Eq. (4.8), we have to take into account the four allowed transitions. We use the notations:

Then:

$$
\begin{aligned}
& \sigma_{j_{0}}(\lambda)=a\left(j_{0}, \lambda+i\right)-a\left(j_{0}, \lambda\right) \\
& \varrho_{\lambda}\left(j_{0}\right)=a\left(j_{0}+1, \lambda\right)-a\left(j_{0}, \lambda\right) .
\end{aligned}
$$

$$
\begin{aligned}
\sigma_{j_{0}}^{2}(\lambda) & B^{\lambda+i}\left(P_{\mu}, P_{\nu}\right)+\sigma_{j_{0}}^{2}(\lambda-i) B^{\lambda-i}\left(P_{\mu}, P_{\nu}\right) \\
& +\varrho_{\lambda}^{2}\left(j_{0}\right) B^{j_{0}+1}\left(P_{\mu}, P_{\nu}\right)+\varrho_{\lambda}^{2}\left(j_{0}-1\right) B^{j_{0}-1}\left(P_{\mu}, P_{v}\right)=-\varepsilon i L_{\mu \nu} .
\end{aligned}
$$

The computations are very analogous to those given in part III (2) and we omit the details. The results are the following:

- From the non diagonal part, one gets that:

either $\sigma_{j_{0}}(\lambda)$ is independent of $j_{0}$ and $\varrho_{\lambda}\left(j_{0}\right)$ independent of $\lambda$

either

$$
\sigma_{j_{0}}(\lambda)=(-)^{j_{0}} \sigma(\lambda)
$$

$$
\varrho_{\lambda}\left(j_{0}\right)=(-)^{i \lambda} \varrho\left(j_{0}\right) \text {. }
$$

- The diagnonal part furnishes 2 equations which can be solved by using the preceding remark. They are:

$$
\begin{aligned}
& j_{0}\left(\alpha_{\lambda-1}^{\prime}-\alpha_{\lambda}^{\prime}\right)+i \lambda\left(\beta_{j_{0}}^{\prime}-\beta_{j_{0}-1}^{\prime}\right)=0 \\
& i \lambda\left(\alpha_{\lambda}^{\prime}-\alpha_{\lambda-1}^{\prime}\right)-\left(\alpha_{\lambda}^{\prime}+\alpha_{\lambda-1}^{\prime}\right)-j_{0}\left(\beta_{j_{0}}^{\prime}-\beta_{j_{0}-1}^{\prime}\right) \\
& \quad-\left(\beta_{j_{0}}^{\prime}+\beta_{j_{0}+1}^{\prime}\right)=-\frac{\varepsilon}{2} \quad \text { with }\left\{\begin{array}{l}
\alpha_{\lambda}^{\prime}=\alpha_{\lambda} \sigma^{2}(\lambda) \\
\beta_{j_{0}}^{\prime}=\beta_{j_{0}} \varrho^{2}\left(j_{0}\right) .
\end{array}\right.
\end{aligned}
$$

The solutions are

and

$$
\sigma^{2}(\lambda)=\frac{\varepsilon \lambda^{2}(\lambda+i)^{2}+a_{1}\left(\lambda^{2}+(\lambda+i)^{2}\right)+b_{1}}{m^{2}(s+i \lambda)(s-i \lambda+1)}
$$

$$
\varrho\left(j_{0}\right)=\sigma\left(-i j_{0}\right) \text {. }
$$

$A$ is an irrational function of the two Casimirs in general. The only simple case can be computed by requiring (4.19) to be a perfect square. The solutions is then unique up to a constant factor, it is:

$$
a(\lambda)= \pm \frac{\sqrt{\varepsilon}}{2 m} i\left(1+\lambda^{2}-j_{0}^{2}\right)
$$

and corresponds here also to the Gell-Mann formula.

Acknowledgements. We thank J. LAScoux for its interest and for a critical reading of the manuscript.

\section{Conclusion}

For all the reasons exposed in I. $5 \mathrm{a}, \mathrm{b}$, c, it is important to know the group $H^{1}(\mathscr{G}, L(V))$ for the deformations of the representations. We have seen that the group $H^{1}(\mathscr{G}, L(V))$ is a one-dimensional space on $C$, both 
for the Euclidian and the Poincare algebras (in the case $m>0, S \geqq 0$ for the last one). With some hermiticity conditions, necessary to produce a deformation into unitary representations of the group, it becomes a one dimensional space on $R$. Then the deformations of Poincaré's representations, the algebra being fixed, correspond simply to a variation of the mass, the spin being rigid. (As it can be expected in general for all the discrete numbers indexing the representation). When the algebra is also deformed, we have seen the general form of the first order deformation, the most simplest example being given by the "Gell Mann Formula".

\section{Appendix}

Deformation of the Continuous Series of $S L(2, R)$

Let us study the first order condition (1.6) (or 2.2) satisfied by a general deformation

$$
\varphi_{t}(x)=\sum_{n=0}^{\infty} \varphi_{n}(x) t^{n}
$$

We start from a unitary representation of the $S L(2, R)$ Lie algebra: [of the class $C_{q}^{0}$ or $C_{q}^{1 / 2}$ in Bargmann notations],

$$
\begin{aligned}
& \varphi_{0}\left(H_{3}\right) f_{m}=m f_{m} \\
& \varphi_{0}\left(H_{+}\right) f_{m}=\alpha_{m} f_{m+1} \\
& \varphi_{0}\left(H_{-}\right) f_{m}=\bar{\alpha}_{m-1} f_{m-1}
\end{aligned}
$$

where $m$ assumes all integers (or half integers) values between $-\infty,+\infty$. (One may choose $\alpha_{m}=(q+m(m-1))^{1 / 2} q$ being the value of the Casimir. We require that the deformation leaves the space $\bigoplus_{-\infty}^{+\infty} f_{m}$ inchanged, and the simplest way is to ask that $\varphi_{0}\left(H_{3}\right)$ remains fixed during the deformation.

From $(2.2 \mathrm{c}), \varphi_{1}\left(H_{ \pm}\right)$transforms under $\varphi_{0}\left(H_{3}\right)$ like $\varphi_{0}\left(H_{ \pm}\right)$, or equivalently:

$$
\left.\begin{array}{l}
\varphi_{1}\left(H_{+}\right) f_{m}=C_{m} f_{m+1} \\
\varphi_{1}\left(H_{-}\right) f_{m}=d_{m} f_{m-1}
\end{array}\right\}
$$

The last relations $(2.2 \mathrm{~b})$ is

i. e.

$$
\left[\varphi_{0}\left(H_{+}\right), \varphi_{1}\left(H_{-}\right)\right]=\left[\varphi_{0}\left(H_{-}\right), \varphi_{1}\left(H_{+}\right)\right]
$$

$$
d_{m} \alpha_{m-1}-\alpha_{m} d_{m+1}=C_{m} \bar{\alpha}_{m}-\bar{\alpha}_{m-1} C_{m-1}
$$

or

$$
d_{m} \alpha_{m-1}+\bar{\alpha}_{m-1} C_{m-1}=C_{m} \bar{\alpha}_{m}+\alpha_{m} d_{m+1}=a,
$$

where $a$ is a complex number independant of $m$. 
The solution can be written:

$$
\left.\begin{array}{rl}
d_{m} & =\frac{1}{\alpha_{m-1}}\left(\frac{a}{2}+\varrho_{m}\right) \\
C_{m-1} & =\frac{1}{\bar{\alpha}_{m-1}}\left(\frac{a}{2}-\varrho_{m}\right) \cdot
\end{array}\right\}
$$

Let us examine now the condition of being a coboundary for $\varphi_{1}$. The general form of a coboundary is of the type A.2 (because we are dealing with $\mathrm{H}_{3}$-relative cohomology), with:

$$
\left.\begin{array}{l}
C_{m}=\left(\mu_{m+1}-\mu_{m}\right) \alpha_{m} \\
d_{m}=\left(\mu_{m-1}-\mu_{m}\right) \bar{\alpha}_{m-1}
\end{array}\right\}
$$

For some arbitrary (complex) numbers $\mu_{m}$.

It is then easy to see that any solution of the form (A.5) must have: $a=0$.

It remains one condition: $\varrho_{n}=\left|\alpha_{m}\right|^{2}\left(\mu_{m-1}-\mu_{m}\right)$ but it is always possible given a number $x_{m}=\frac{\varrho_{m}}{\left|\alpha_{m}\right|^{2}}$ to construct such $\mu_{n}$. It is enough to take:

$$
\mu_{0}=0, \mu_{n}=\sum_{p=1}^{n} x_{p} \quad \mu_{-n}=-\sum_{p=0}^{n-1} x_{-p} .
$$

To sum up, the group $H^{1}\left(S L(2, R), H_{3}, L(V)\right)$ (relative to $\left.H_{3}\right)$ is - on $C$, a one-dimensional group, a representative of which being given by:

$$
\begin{aligned}
& \varphi_{1}\left(H_{+}\right) f_{m}=\frac{1}{\bar{\alpha}_{m}} f_{m+1} \\
& \varphi_{1}\left(H_{-}\right) f_{m}=\frac{1}{\alpha_{m-1}} f_{m-1} .
\end{aligned}
$$

One can check that there does not exist a non trivial deformation only at the first order. Nevertheless it is easy to prove that a cocycle (A.6) is "integrable" (i. e. is the first term of a family $\varphi_{n}$ given a deformation). It is enough to consider the representation corresponding to the value $q+$ at of the casimir. One has (up to a phase)

$$
\begin{aligned}
& C_{m}(t)=(q+m(m-1)+a t)^{1 / 2} \\
& C_{m}(t)=\alpha_{m}+\frac{1}{2} \frac{a t}{\alpha_{m}}+\cdots .
\end{aligned}
$$

It is also easy to measure the weakness of the "equivalence to the first order" on that example. Using the previous deformation, one can construct a series of non equivalent deformations with the same first term $\varphi_{1}$.

\section{Restricted Deformations}

Until now, we have not taken into account any consideration of unitarity, and a complex number $a$ leads to a non-unitary deformed representation.

18 Commun. math. Phys., Vol. 9 
Let us require now the unitarity of the deformation. For $\varphi_{1}$, it means : $d_{m}=\bar{C}_{m-1}$ and from (A.3) one sees that $a$ is then a real number.

In that case (choosing a real $\alpha_{m}$ ), $H^{1}$ is a one-dimensional space, on $R$. Both cases correspond well to the intuitive picture.

Since the character integer or half integer is not altered by a deformation, we can choose for "structure space" the complex plane: a representation being determined by the value of its casimir $q$. The unitary continuous series are lying on an axis: $q \geqq 1 / 4$ so that:

- a deformation into a unitary representations can start into only one direction (this axis);

- a deformation into a non unitary one can start into any directions in the complex plane: this is expressed by a multiplication by a complex number.

\section{References}

1. George, C., and M. Levy-Nahas: J. Math. Phys. 7, 980 (1966).

2. Levy-Nahas, M.: To be published (in french) in the Compte-rendu du Colloque de Gif-sur-Yvette.

3. Hermann, R.: Analytic continuation of group representations: III. Commun. Math. Phys. 3, 75 (1966); IV. Commun. Math. Phys. 5, 131 (1967).

4. Gerstenhaber, M.: Ann. Math. 78, 267 (1963).

5. Hermann, R.: II. Commun. Math. Phys. 3, 53 (1966).

6. Levy-Nahas, M.: J. Math. Phys. 8, (1967).

7. Séminaire "Sophus Lie" 1954/1955. E. N. S. Paris.

8. George, C.: Exposé à l'Inst. Henri Poincaré en 1966 (Paris).

9. Hochschild, G., and J. P. Serre: Ann. Math. 57, 591 (1953).

10. Chakrabarti, A., M. Levy-Nahas et R. Seneor: Preprint, École Polytechnique, Paris, Juin 1967.

11. Mukunda, N.: Preprint, Syracuse Univ. NYO-3399-107 (1967).

12. Chakrabarti, A.: J. Math. Phys. 3, 405 (1962).

13. Behrend: On the reversal problem. Math. Inst. Freie Univ. Berlin (1966).

14. Hermann, R.: Commun. Math. Phys. 2, 155 (1966); and references quoted of therein (Lie groups for physicists).

15. Fronsdal, C.: Rev. Mod. Phys. 37, 221 (1965).

16. Gotsman, E., and Y. NE'eman: Coral Gables Conf. Jan. 1966, p. 137.

17. Barut, A. O., and A. Böнm: Phys. Rev. 139, B 1107 (1965).

18. Hermann, R.: V. Commun. Math. Phys. 5, 157 (1967); VI. Preprint, Stanford Univ. California, March 1967.

Dr. R. Seneor

Centre de Physique Théorique de

l'Ecole Polytechnique

17, Rue Descartes

F-75 Paris V 